\section{Efficacy of an Automated Multiple Emitter Whole-Room Ultraviolet-C Disinfection System Against Coronaviruses MHV and MERS-CoV}

\author{
Kurt Bedell, BS; ${ }^{1}$ Adam H. Buchaklian, $\mathrm{PhD} ;^{2}$ \\ Stanley Perlman, MD, $\mathrm{PhD}^{1,3}$
}

Efficient and automated methods of disinfecting surfaces contaminated with the Middle Eastern respiratory syndrome coronavirus (MERS$\mathrm{CoV}$ ) may prevent the spread of the virus. Here we report the efficacy and use of an automated triple-emitter whole room UV-C disinfection system to inactivate mouse hepatitis virus, strain A59 (MHV-A59) and MERS-CoV viruses on surfaces with a $>5 \log _{10}$ reduction.

Infect Control Hosp Epidemiol 2016;37:598-599

The virus responsible for Middle Eastern respiratory syndrome (MERS), MERS-coronavirus ( $\mathrm{CoV}$ ) is a lineage $\mathrm{C} \beta$-coronavirus similar to the lineage $\mathrm{A}$ murine $\beta$-coronavirus, mouse hepatitis virus, strain A59 (MHV-A59). Coronaviruses were first identified as the causative agent of the severe acute respiratory syndrome (SARS) in 2002 in China, and the Middle Eastern respiratory syndrome (MERS) in the Middle East in 2012. ${ }^{1-3}$ The first reported case of MERS occurred in Saudi Arabia in 2012 and resulted in 76 deaths. ${ }^{4}$ Since that time, cases of MERS have continued to occur in both the Middle East as well as in other countries in Europe, Asia, Africa, and North America. A recent outbreak, involving more than 180 patients, occurred in South Korea. ${ }^{5}$ MERS has a reported mortality rate of approximately $36 \% ;, 3,6$ it is thought to be transmitted from camels to humans through direct contact; however, obvious camel-to-human interaction has not been documented in all cases. Human-tohuman transmission has also been identified in hospital and household transmission during MERS outbreaks. The ability of coronaviruses to rapidly mutate increases the risk of a large-scale outbreak or epidemic in the future. ${ }^{2}$ MHV-A59 is structurally similar to the MERS-CoV but causes hepatic and neuronal disease in mice. The MHV-A59 virus is an ideal model virus with which to study the effects of UV-C against MERS-CoV because it has many similarities to the human virus but can be handled at the biosafety level 2 (BSL2) laboratory. Along with studies of MHV-A59, the Surfacide multiple-emitter system (Surfacide, Naperville, IL) was also tested against the MERS-CoV virus.

\section{METHODS}

The Surfacide multiple-emitter automated UV-C disinfection system consists of 3 ultraviolet C (UV-C) emitters with a diameter of $59 \mathrm{~cm}$ and a height of $195 \mathrm{~cm}$. All systems include both a touch screen control tablet and a motion sensor that double as jointers to connect the emitters together for transport. The system utilizes a laser to identify the size of the disinfection space and to identify near-field objects. Based on these measurements the system then calculates the disinfection cycle time accordingly while rotating $360^{\circ}$.

Triplicate $10-\mu \mathrm{L}$ aliquots of MHV-A59 were loaded onto sterile glass coverslips at a concentration of $1.6 \mathrm{e} 8 \mathrm{pfu} / \mathrm{mL}$ and allowed to dry. Coverslips containing dried virus were then placed in a UV transparent Petri dish and either left untreated or exposed to UV-C radiation from the Surfacide system placed 4 feet $(1.22 \mathrm{~m})$ from the samples for predetermined times between 0 and 60 minutes. The coverslips containing dried virus were then placed in Dulbecco's Modified Eagle's Medium (DMEM) media on ice to resuspend the virus. Serial 1:10 dilutions were inoculated onto Hela cells expressing the MHV receptor ${ }^{7}$ and allowed to incubate for 45 minutes at $37^{\circ} \mathrm{C}$. Medium containing virus was removed and the cells were allowed to incubate overnight in D10 media. Plaque counts were determined the following day by combining $1 \%$ neutral red with $2 \times$ media plus agarose and incubating the cells for approximately 3 hours. All studies were conducted in triplicate with replicate experiments.

Testing MERS-CoV sensitivity to UV-C was conducted under strict containment BSL3 laboratory protocols due to the infective nature of the virus, and all steps were carried out above bleachsoaked pads to prevent virus spread. The moist environment created by the bleach soaked pads in the biosafety cabinet prevented drying of the virus onto glass coverslips. MERS-CoV was therefore loaded onto glass coverslips as small droplets at a concentration of $8.9 \mathrm{e} 5 \mathrm{pfu} / \mathrm{mL}$ in UV-C permeable dishes and exposed to UV-C energy from the Surfacide emitter placed 4 feet $(1.22 \mathrm{~m})$ from the dishes. Samples were removed at several time points between 0 and 30 minutes. Virus resuspension and dilution were carried out as described above. Virus dilutions were placed onto $90 \%$ confluent African green monkey kidney cell-line (Vero81) cells at $37^{\circ} \mathrm{C}$ for 40 minutes. Following a 40 -minute incubation, medium containing virus was removed and replaced with $2 \times$ medium plus $1.2 \%$ agarose and allowed to solidify. DMEM was then layered on top to prevent drying, and the plates were incubated at $37^{\circ} \mathrm{C}$ for 3 days to allow plaque formation. On day 3 , formalin was added to fix the cells for 20 minutes. Formalin, media, and agarose were then removed and replaced with $0.1 \%$ crystal violet for a 5-minute incubation. This procedure was followed by a phosphate-buffered saline (PBS) wash prior to counting plaques. Again, all samples were prepared in triplicate.

\section{RESULT S}

MHV-A59 dried and MERS-CoV droplets were stable on glass coverslips for at least 60 and 30 minutes, 0.09 and $0.22 \log$ reduction, respectively. Plaque counts indicated that 


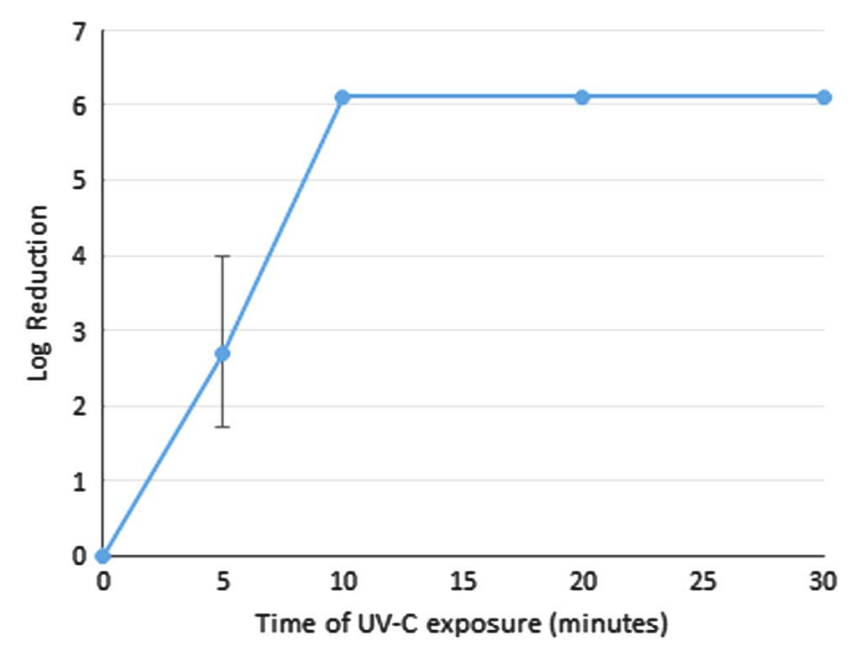

FIGURE 1. Log reduction of MHV-A59 following Surfacide UV-C exposure at a 4 -foot distance $(1.22 \mathrm{~m})$. Virus was reduced by $2.71 \log _{10}$ in 5 minutes and by $6.11 \log _{10}$ in 10 minutes to undetectable levels. Results are the means of triplicate samples.

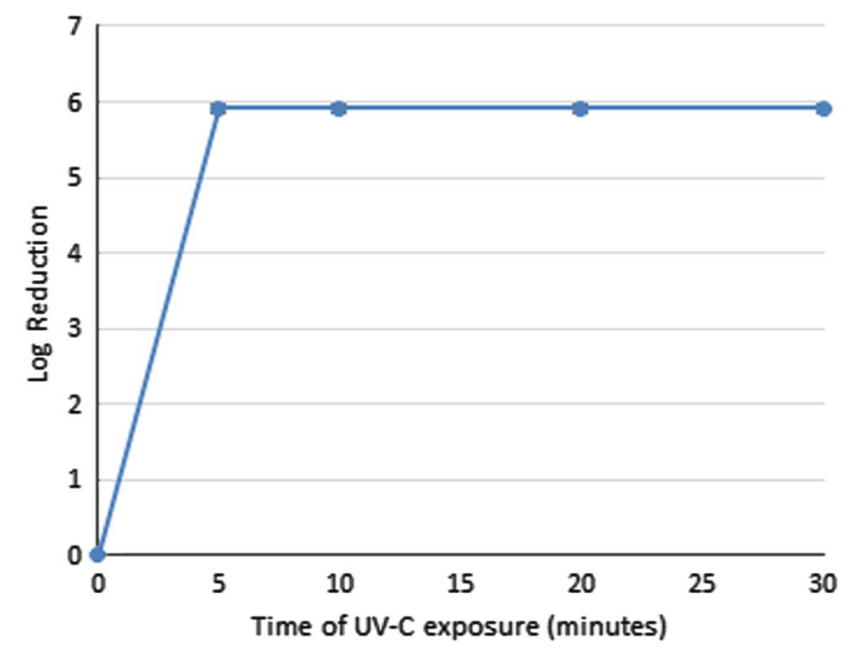

FIGURE 2. Log reduction of MERS-CoV during exposure to Surfacide UV-C at 4 feet $(1.22 \mathrm{~m})$. Log reduction of MERS-CoV on glass coverslips following Surfacide UV-C exposure at a 4 -foot distance. Virus was reduced to $5.91 \log _{10}$ in 5 minutes to undetectable levels. Results are the means of triplicate samples.

the UV-C energy emitted from the Surfacide disinfection system was able to reduce the viral titers of MHV-A59 by an average of $2.71 \log _{10}$ in 5 minutes and $6.11 \log _{10}$ in 10 minutes of exposure, resulting in undetectable levels of MHV-A59 virus (Figure 1). For MERS-CoV, a UV-C exposure time of only 5 minutes resulted in undetectable virus levels that remained undetectable following 30 minutes of total exposure for a $5.9 \log _{10}$ reduction (Figure 2 ).

\section{I S C U S S I O N}

The multiple-emitter continuous UV-C disinfection system was $>99.999 \%$ effective against MHV-A59, a mouse analog of
MERS-CoV, and SARS-CoV in 10 minutes. Applying those same studies to droplets of MERS-CoV resulted in undetectable levels of MERS-CoV virus after only 5 minutes of exposure to the UV-C emitter or a percent reduction of $>99.999 \%$. Notably, UV-C disinfection is most effective when used as an adjunct to standard terminal cleaning of hard surfaces and should not be used to replace good cleaning practices. ${ }^{8}$ This study is the first to document the effectiveness of a multiple-emitter, automated, whole-room UV-C system or any whole-room disinfection system against RNA viruses like MHV-A59, MERS-CoV, and SARS-CoV. The use of the Surfacide whole-room UV-C disinfection system during MERS outbreaks may prevent the nosocomial spread of the virus and protect staff in the process.

\section{ACKNOWLEDGMENTS}

Adam Buchaklian, PhD, is the Director of Research for Surfacide, LLC, and provided the UV-C disinfection system as well as recommendations on study design. All studies were conducted independently in the laboratory of Dr. Stanley Perlman at the University of Iowa. All other authors report no conflict of interest.

Affiliations: 1. University of Iowa, Department of Microbiology, Iowa City, Iowa; 2. Surfacide LLC, Waukesha, Wisconsin; 3. University of Iowa, Department of Pediatrics, Iowa City, Iowa.

Address correspondence to Adam Buchaklian, PhD, Surfacide, LLC, 407 Pilot Ct., Waukesha, WI 53188 (adam.buchaklian@surfacide.com).

Received October 19, 2015; accepted December 23, 2015; electronically published January 28, 2016

(C) 2016 by The Society for Healthcare Epidemiology of America. All rights reserved. 0899-823X/2016/3705-0015. DOI: 10.1017/ice.2015.348

\section{REFERENCES}

1. Nie QH, Luo XD, Hui WL. Advances in clinical diagnosis and treatment of severe acute respiratory syndrome. World $J$ Gastroenterol 2003;9:1139-1143.

2. de Groot RJ, Baker SC, Baric RS, et al. Middle East respiratory syndrome coronavirus (MERS-CoV); announcement of the Coronavirus Study Group. J Virol 2013;5:13-15.

3. Zumla A, Hui DS, Perlman S. Middle East respiratory syndrome. Lancet 2015;386:995-1007.

4. Zaki AM, van Boheemen S, Bestebroer TM, Osterhaus ADME, Fouchier RAM. Isolation of a novel coronavirus from a man with pneumonia in Saudi Arabia. N Engl J Med 2012;367:1814-1820.

5. Khan A, Farooqui A, Guan Y, Kelvin DJ. Lessons to learn from MERS-CoV outbreak in South Korea. J Infect Dev Ctries 2015;9:7-10.

6. Memish ZA, Al-Tawfiq JA, Alhakeem RF, et al. Middle East respiratory syndrome coronavirus (MERS-CoV): a cluster analysis with implications for global management of suspected cases. Travel Med Infect Dis 2015;13:311-314.

7. Williams RK, Jiang GS, Holmes K V. Receptor for mouse hepatitis virus is a member of the carcinoembryonic antigen family of glycoproteins. PNAS 1991;88:5533-5536.

8. Anderson DJ, Gergen MF, Smathers E, et al. Decontamination of targeted pathogens from patient rooms using an automated ultraviolet-C-emitting device. Infect Control Hosp Epidemiol 2013;34:466-471. 